\title{
Acute coronary syndrome in patients with prior coronary artery bypass graft: clinical, angiographic profiles and management practices
}

\author{
Authors: Fathima Aaysha Cader, ${ }^{\mathrm{A}}$ Muhammad Maksumul Haq ${ }^{\mathrm{A}}$ and Saidur Rahman Khan ${ }^{\mathrm{A}}$
}

\section{Introduction}

Acute coronary syndrome (ACS) among patients with postcoronary artery bypass graft (CABG) is not uncommonly encountered in current clinical practice. This study aimed to investigate the clinical and angiographic profiles and management strategies among post-CABG patients presenting with ACS at a tertiary care cardiac centre in Dhaka, Bangladesh.

\section{Materials and methods}

This retrospective observational study included all patients with ACS with a prior history of CABG presenting to our institute and undergoing coronary angiography from February 2015 to October 2019. Data were collected from hospital discharge records and the catheterisation laboratory database and statistically analysed.

\section{Results and discussion}

From a total of 591 post-CABG patients who underwent coronary angiography, 223 (37.7\%) presented with ACS. The mean age was $62.15 \pm 8.28$ years and $90.6 \%$ of the patients were male. The majority of post-CABG ACS patients presented with non-ST segment elevation myocardial infarction (NSTEMI; 61.9\%), with lower proportions presenting with unstable angina (32.7\%) and STEMI (5.4\%). Male patients most frequently presented with NSTEMI (63.9\%), while most female patients presented with unstable angina (52.4\%). Overall, 177 (79.9\%) of ACS patients were hypertensive, 109 (48.9\%) dyslipidaemic, 156 (70\%) diabetic, 45 (20.2\%) had chronic kidney disease (CKD) and $111(49.8 \%)$ were smokers. Troponin I levels were elevated in $143(64.1 \%)$. Mean left ventricular ejection fraction was $48.31 \pm 9.48 \%$. Graft vessel disease was identified as culprit in $124(60.2 \%)$ of cases and native artery in 51 (24.8\%). Among

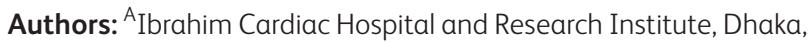
Bangladesh those with graft vessel disease, $129(58.1 \%)$ had saphenous venous graft (SVG) lesions, 12 (5.4\%) had arterial graft lesions and $15(6.8 \%)$ had both arterial and venous graft disease. The majority of patients $(n=143 ; 64.1 \%)$ underwent angiography and graft study by left radial approach, followed by right radial $(24.2 \%)$, right femoral $(11.2 \%)$ and left ulnar approach $(0.4 \%)$. A trend towards more radial intervention was observed as the years progressed. In terms of management strategy, two-thirds of the patients $(n=148 ; 66.7 \%)$ were recommended revascularisation by percutaneous coronary intervention (PCI), 69 (31.1\%) were treated with optimal medical therapy and five $(2.3 \%)$ were referred to have CABG carried out again. In total, 93 (41.9\%) of patients underwent PCI, of which $82.8 \%$ were ad hoc procedures and $17.2 \%$ were elective procedures done during the index admission. The PCI target was a native coronary artery in $53.8 \%$ and a bypass graft in $37.6 \%$. In $8.6 \%$, PCI was performed to both a native coronary and a graft.

Our study is the largest to date to analyse the management practices of patients with prior CABG who subsequently presented with ACS in Bangladesh. Despite an ongoing debate over the best vascular access site for post-CABG intervention, ${ }^{1}$ we found that the left radial approach is reasonable, particularly with experienced operators. As previously noted, ${ }^{1-3}$ saphenous venous grafts are notorious to occlusion, with high rates of restenosis. Data and guidelines recommend native artery revascularisation over graft vessel intervention, a practice that was appropriately observed in this study. ${ }^{3,4}$

\section{Conclusion}

The most common ACS presentation post-CABG patient population was NSTEMI, although women presented more frequently with unstable angina. Venous grafts were the predominant culprit vessel. PCI remains the most frequently utilised strategy for revascularisation, with native artery PCI taking precedence over grafts.

\section{Conflicts of interest}

None declared. 


\section{References}

1 Xenogiannis I, Tajti P, Hall AB et al. Update on cardiac catheterization in patients with prior coronary artery bypass graft surgery. JACC Cardiovasc Interv 2019:12;1635-49.

2 Goldman S, Zadina K, Moritz T et al. Long-term patency of saphenous vein and left internal mammary artery grafts after coronary artery bypass surgery: results from a Department of Veterans Affairs Cooperative Study. J Am Coll Cardiol 2004;44:2149-56.
3 Brilakis ES, Rao SV, Banerjee S et al. Percutaneous coronary intervention in native arteries versus bypass grafts in prior coronary artery bypass grafting patients: a report from the National Cardiovascular Data Registry. JACC Cardiovasc Interv 2011;4:844-50.

4 Neumann FJ, Sousa-Uva M, Ahlsson A et al. ESC Scientific Document Group. 2018 ESC/EACTS Guidelines on myocardial revascularization. Eur Heart J 2018:40; 87-165. 